\title{
Driving Strategy Selection for Cooperative Vehicles using Maneuver Templates
}

\author{
Stefanie Manzinger ${ }^{1}$, Marion Leibold ${ }^{2}$, and Matthias Althoff ${ }^{1}$
}

\begin{abstract}
We introduce a novel concept based on maneuver templates, which are formalized collaborative maneuvers, to select cooperative driving strategies. The approach is based on the exclusion principle, where we derive provable conditions to discard unsafe cooperative maneuvers for a given traffic situation. We thereby consider the full action set of all cooperative vehicles and do not discretize the action space. We demonstrate the applicability of our approach with numerical examples.
\end{abstract}

\section{INTRODUCTION}

Collaborative automated vehicles will contribute significantly to improving traffic flow and safety. A well-known method for increasing traffic throughput with concurrent reduction of fuel consumption is platooning, which is predominantly a control problem, see e.g. [1], [2]. Additionally, there exist variational techniques, e.g. [3]-[5], and graphbased techniques, e.g. [6], for planning even more complex coordinated motions. While numerical optimization offers the possibility of designing trajectories according to a cost function, it often suffers from high computational complexity. This is usually tackled by simplifying the system dynamics, see e.g. [5]. Furthermore, there is often the need for meaningful initialization, see e.g. [3], and the set of vehicles forming a collaborative group may be required, see e.g. [7]. In the case of graph-based techniques, only a small fraction of admissible actions can be considered, since one obtains $n^{m}$ possible plans for $m$ vehicles with $n$ admissible actions.

The current literature shows that many promising approaches exist. However, we believe that the efficiency of motion planning algorithms can be increased by introducing methods for selecting cooperative driving strategies. Several approaches have been presented which address specific traffic situations, e.g. the coordination of multiple vehicles at road intersections [8]-[11], lane-changing and merging [12], and lane closures [13]. Furthermore, decision-making techniques based on algorithms from game theory, e.g. auctions, have received major attention as a way to negotiate cooperative driving strategies among several automated vehicles [14][17]. In [18], a holistic approach is proposed which calculates a high-level strategy as well as a low-level control strategy

\footnotetext{
*This work was supported by the Deutsche Forschungsgemeinschaft (German Research Foundation) within the Priority Program SPP 1835 Cooperative Interacting Automobiles (grant number: AL 1185/4-1)

${ }^{1}$ Stefanie Manzinger and Matthias Althoff are with the Department of Informatics, Technical University of Munich, 85748 Garching bei München, Germany $\{$ stefanie.manzinger, althoff\}ein.tum.de ${ }^{2}$ Marion Leibold is with the Chair of Automatic Control Engineering, Technical University of Munich, Theresienstr. 90, 80333 München, Germany \{marion.leibold\}etum.de
}

simultaneously on the basis of reachable sets and risk assessment. The work of [19] proposes a high-level maneuver planner for cooperative vehicles on highways based on Monte Carlo tree search.

The focus of most work for cooperative strategy planning is the optimization of various objectives, e.g. traffic flow and passenger comfort, which is of major importance in nonhazardous traffic situations. In emergency situations however, such objectives are subordinate, as the main goal is to quickly find a collision-free maneuver. Therefore, we introduce a novel concept for discarding unsafe collaborative driving strategies, with an emphasis on emergency situations. Our approach is based on the exclusion principle, through which we prove that certain maneuvers are not safe given the current traffic situation. For instance, we can verify that in a certain situation, it is not possible to perform a collaborative merging maneuver, but a collaborative braking maneuver can be done. We refer to the latter high-level maneuvers as maneuver templates. Our approach does not replace a trajectory planning module; however, it can be combined with any motion planner.

\section{Problem Statement and Methodology}

We formalize cooperative driving strategies by using maneuver templates, which are composed of a model $M$ and a set of constraints $\mathcal{C}$. Choosing different sets of constraints $\mathcal{C}$ results in various cooperation strategies.

Definition 1 (Maneuver Template): Let us represent a maneuver template $T$ as a tuple $T=(M, \mathcal{C})$ of a model $M$ and a set of constraints $\mathcal{C}$ :

- A model $M$ itself is modeled as a tuple $M=\left(f, \mathcal{Z}_{0}, \mathcal{U}\right)$. Therein, $f$ is the right-hand side of a differential equation $\dot{\mathbf{z}}(t)=f(\mathbf{z}(t), \mathbf{u}(t))$ describing the continuous dynamics of several traffic participants, where $\mathbf{z}$ is the state, $\mathbf{u}$ is the input, and $t$ is the time. The possible initial states and the inputs are bounded by sets: $\mathbf{z}_{0} \in$ $\mathcal{Z}_{0} \subset \mathbb{R}^{n}, \forall t: \mathbf{u}(t) \in \mathcal{U} \subset \mathbb{R}^{m}$.

- The discrete set $\mathcal{C}=\left\{C_{1}(\mathbf{z}, \mathbf{u}), \ldots, C_{p}(\mathbf{z}, \mathbf{u})\right\}$ contains individual constraints $C_{i}(\mathbf{z}, \mathbf{u}): \mathbb{R}^{n} \times \mathbb{R}^{m} \rightarrow$ \{true,false $\}$ that evaluate to true or false given the state and input.

We further introduce the solution of the differential equation $\dot{\mathbf{z}}(t)=f(\mathbf{z}(t), \mathbf{u}(t))$ as $\chi\left(t ; \mathbf{z}_{0}, \mathbf{u}(\cdot)\right)$, where $\mathbf{u}(\cdot)$ refers to the input trajectory. The set of feasible solutions that is represented by a maneuver template is

$$
\begin{aligned}
\mathcal{Z}(t)= & \left\{\chi\left(t ; \mathbf{z}_{0}, \mathbf{u}(\cdot)\right) \mid \mathbf{z}_{0} \in \mathcal{Z}_{0}, \forall t: \mathbf{u}(t) \in \mathcal{U}\right. \\
& \left.\forall t \forall i: C_{i}\left(\chi\left(t ; \mathbf{z}_{0}, \mathbf{u}(\cdot)\right), \mathbf{u}(t)\right)=\text { true }\right\}
\end{aligned}
$$


The goal of our approach is to check if $\mathcal{Z}(t)=\emptyset$ holds for a given model $M$ with constraints $\mathcal{C}$ and initial state $\mathbf{z}_{0}$. This means that a driving strategy corresponding to maneuver template $T$ is not feasible and we can discard maneuver template $T$.

In this paper, we use optimal control theory to determine $\mathcal{Z}(t)$. Let us introduce maneuver template $T$ with the discrete set of constraints $\mathcal{C}=\mathcal{B} \cup \mathcal{H} \cup \mathcal{A}$, which is composed of the set of initial constraints $\mathcal{B}=$ $\left\{b_{1}\left(\mathbf{z}_{0}, t_{0}\right), b_{2}\left(\mathbf{z}_{0}, t_{0}\right), \ldots\right\}$, the set of state constraints $\mathcal{H}=$ $\left\{h_{1}(\mathbf{z}(t), t), h_{2}(\mathbf{z}(t), t), \ldots\right\}$, and the set of terminal constraints $\mathcal{A}=\left\{a_{1}\left(\mathbf{z}\left(t_{f}\right), t_{f}\right), a_{2}\left(\mathbf{z}\left(t_{f}\right), t_{f}\right), \ldots\right\}$. We introduce the operator $\circ \in\{\leq,<,=\}$ and reformulate (1) such that $\mathcal{Z}(t)$ is the solution of the following boundary-value problem:

$$
\begin{gathered}
\dot{\mathbf{z}}(t)=f(\mathbf{z}(t), \mathbf{u}(t)), \mathbf{z}_{0} \in \mathcal{Z}_{0}, \mathbf{u}(t) \in \mathcal{U} \\
\forall k: b_{k}\left(\mathbf{z}_{0}, t_{0}\right) \circ 0, \\
\forall i: a_{i}\left(\mathbf{z}\left(t_{f}\right), t_{f}\right) \circ 0, \\
\forall t \forall j: h_{j}(\mathbf{z}(t), t) \circ 0 .
\end{gathered}
$$

Additionally, the following cost function is introduced:

$$
J\left(\mathbf{z}(t), \mathbf{u}(t), t_{f}\right)=S\left(\mathbf{z}\left(t_{f}\right), t_{f}\right)+\int_{t_{0}}^{t_{f}} F(\mathbf{z}(t), \mathbf{u}(t), t) \mathrm{d} t,
$$

with terminal costs $S$ and running costs $F$. We overapproximate the set of solutions $\mathcal{Z}(t)$ to facilitate its computation. If we obtain a new model $M^{\prime}$ with constraints $\mathcal{C}^{\prime} \subseteq \mathcal{C}$ and set $\mathcal{Z}^{\prime}(t)$ by relaxing the constraints on model $M$ or by removing constraints $C_{i}(\mathbf{z}, \mathbf{u})$ from $\mathcal{C}$, then $\mathcal{Z}(t) \subseteq \mathcal{Z}^{\prime}(t)$ holds [20]. The overapproximation implies that $\mathcal{Z}^{\prime}(t)=$ $\emptyset \Rightarrow \mathcal{Z}(t)=\emptyset$ holds; therefore, maneuver templates can be discarded despite the overapproximation of $\mathcal{Z}(t)$.

\section{Driving Strategy Selection With Maneuver TEMPLATES}

Let us introduce the traffic scene illustrated in Fig. 1 to explain the general concept of maneuver templates: Vehicle $V_{1}$ intends to avoid obstacle $O_{1}$ in collaboration with vehicles $V_{2}$ and $V_{3}$. Four offline generated maneuver templates, $T_{A}$, $T_{B}, T_{C}$, and $T_{D}$, are available, wherein each maneuver template represents a different cooperative driving strategy. In order to select a collaborative driving strategy, three steps are executed: Matching, Feasibility, and Selection.

a) Matching: First, it is determined if the current traffic situation matches the admissible initial constraints $b_{k}\left(\mathbf{z}_{0}, t_{0}\right)$ of the maneuver templates. Considering the example scenario, maneuver templates $T_{A}, T_{B}$, and $T_{C}$ match the current traffic situation, whereas maneuver template $T_{D}$ is rejected since the number of collaborative vehicles does not coincide with the current traffic situation.

b) Feasibility: Second, the solution set $\mathcal{Z}(t)$ of each maneuver template is determined. Maneuver templates, for which $\mathcal{Z}(t)=\emptyset$ holds, are removed. As depicted in Fig. 1 , maneuver template $T_{B}$ is rejected, since vehicle $V_{2}$ is not able to decelerate sufficiently such that vehicle $V_{1}$ can merge in front of it.

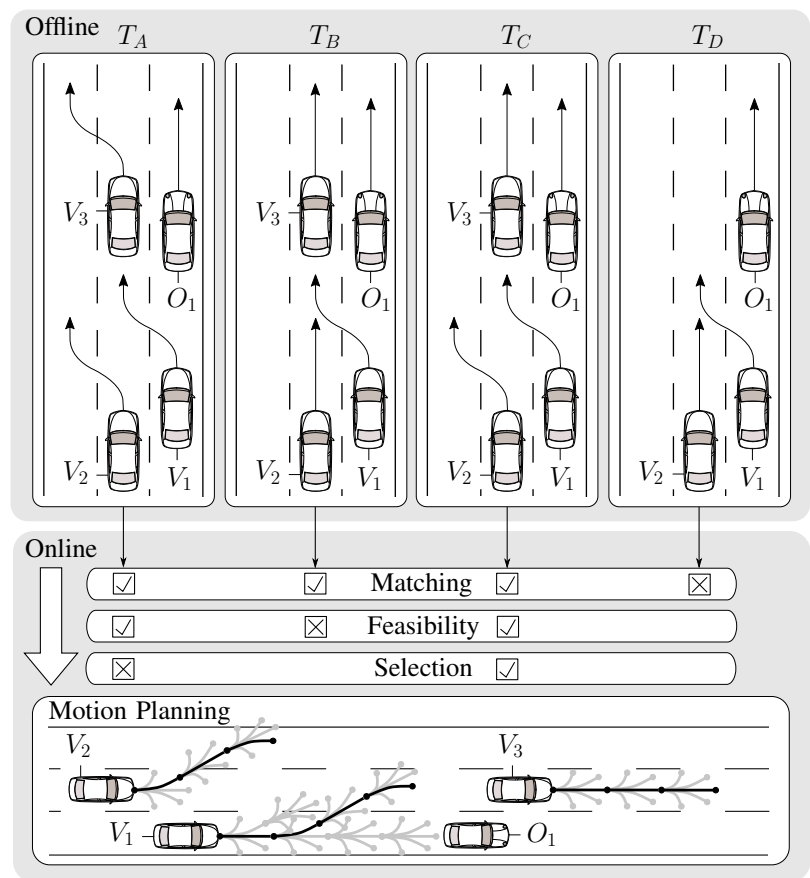

Fig. 1: Overview of motion planning with maneuver templates.

c) Selection: Next, the remaining maneuver templates are assessed in order to rank the most promising driving strategies. The solution set $\mathcal{Z}(t)$ of the maneuver template with the lowest cost can be forwarded to the motion planner. As visualized in Fig. 1, maneuver template $T_{C}$ is selected for guiding the trajectory planner, where vehicles $V_{1}$ and $V_{2}$ perform a lane change maneuver and vehicle $V_{3}$ accelerates to open a gap.

It cannot be guaranteed that there will be a suitable maneuver template available for every possible traffic situation. In the event that there does not exist any suitable maneuver template, the motion planning algorithm is executed without the support of maneuver templates. However, vehicles usually move in semi-structured environments shaped by traffic rules; thus, various traffic flow patterns reoccur frequently. Zhang et al. [21] found that a small number of traffic patterns suffices to comprise the majority of traffic scenarios at signalized intersections. This indicates that a reasonable number of maneuver templates can cover a wide range of traffic scenarios. It should be noted that our method can be applied as soon as the database contains a single maneuver template only. Since novel maneuver templates can be added step by step, this facilitates the development process.

Our focus in this paper is on the construction of maneuver templates and the determination of their solution set $\mathcal{Z}(t)$ (see Fig. 1). Using optimal control theory, we provide conditions to determine if $\mathcal{Z}(t)$ is empty based on models that are subsequently introduced.

\section{Vehicle And Obstacle Models}

We intentionally limit our approach to maneuver templates on straight roads, where non-cooperative vehicles acting as obstacles keep their lane in order to focus on the novel 


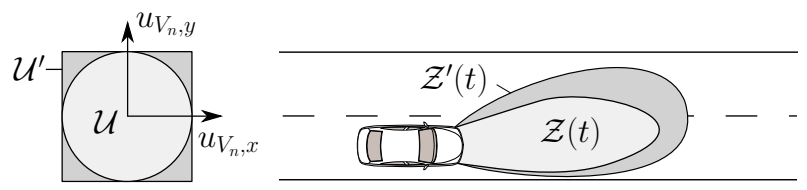

Fig. 2: Enlargement of solution set $\mathcal{Z}(t)$ by overapproximating the friction circle.

aspects of maneuver templates. It should be noted that our approach is applicable to different obstacle or road models. Furthermore, we neglect the spatial extension of the traffic participants and describe the cooperative and non-cooperative vehicles as point masses. This leads to an overapproximation of the solution set $\mathcal{Z}(t)$.

\section{A. Cooperative Vehicles}

As pointed out in Sec. II, we relax the kinematic constraints of the vehicle model in order to facilitate the computation of $\mathcal{Z}(t)$. To this end, we model the dynamics of the $n$-th cooperative vehicle with two double integrators:

$$
\begin{array}{ll}
\dot{s}_{V_{n}, x}(t)=v_{V_{n}, x}(t), & \dot{v}_{V_{n}, x}(t)=u_{V_{n}, x}(t), \\
\dot{s}_{V_{n}, y}(t)=v_{V_{n}, y}(t), & \dot{v}_{V_{n}, y}(t)=u_{V_{n}, y}(t),
\end{array}
$$

with position $\left(s_{V_{n}, x}, s_{V_{n}, y}\right)$, velocity $\left(v_{V_{n}, x}, v_{V_{n}, y}\right)$, and input $\left(u_{V_{n}, x}, u_{V_{n}, y}\right)$. We decouple the longitudinal and lateral dynamics by overapproximating the friction circle with an axis-aligned box (see Fig. 2):

$$
\begin{aligned}
\mathcal{U}^{\prime}=\left\{\left[u_{V_{n}, x}(t), u_{V_{n}, y}(t)\right] \in \mathbb{R}^{2} \mid \underline{u}_{V_{n}, x}\right. & \leq u_{V_{n}, x}(t) \leq \bar{u}_{V_{n}, x} \wedge \\
\underline{u}_{V_{n}, y} & \left.\leq u_{V_{n}, y}(t) \leq \bar{u}_{V_{n}, y}\right\},
\end{aligned}
$$

with $\underline{u}_{V_{n}, x}, \underline{u}_{V_{n}, y} \in \mathbb{R}^{<0}$ and $\bar{u}_{V_{n}, x}, \bar{u}_{V_{n}, y} \in \mathbb{R}^{>0}$. The joint dynamics of $N$ cooperative vehicles is

$$
\frac{\mathrm{d}}{\mathrm{d} t} \underbrace{\left[\begin{array}{l}
\mathbf{x}(t) \\
\mathbf{y}(t)
\end{array}\right]}_{\mathbf{z}(t)}=\left[\begin{array}{cc}
\mathbf{A} & \mathbf{0} \\
\mathbf{0} & \mathbf{A}
\end{array}\right] \mathbf{z}(t)+\left[\begin{array}{cc}
\mathbf{B} & \mathbf{0} \\
\mathbf{0} & \mathbf{B}
\end{array}\right] \underbrace{\left[\begin{array}{l}
\mathbf{u}_{x}(t) \\
\mathbf{u}_{y}(t)
\end{array}\right]}_{\mathbf{u}(t)},
$$

with system matrix $\mathbf{A}$ and input matrix $\mathbf{B}$ obtained from (2), state $\mathbf{x}(t)=\left[s_{V_{1}, x}, v_{V_{1}, x}, \ldots, s_{V_{N}, x}, v_{V_{N}, x}\right]^{T}$, and input $\mathbf{u}_{x}(t)=\left[u_{V_{1}, x}, \ldots, u_{V_{N}, x}\right]^{T}$. The state $\mathbf{y}(t)$ and input $\mathbf{u}_{y}(t)$ are defined likewise.

\section{B. Non-Cooperative Vehicles}

In this paper, the dynamics of the $j$-th non-cooperative vehicle acting as an obstacle is modeled as a double integrator with constant acceleration $\left(u_{O_{j}, x}=\right.$ const.). We thereby take into account that obstacles do not accelerate backwards once they have stopped. The resulting longitudinal dynamics of the $j$-th obstacle is

$$
\begin{aligned}
& \forall u_{O_{j}, x} \in \mathbb{R}^{<0}: \\
& s_{O_{j}, x}(t)= \begin{cases}s_{O_{j}, x}\left(t_{0}\right)+v_{O_{j}, x}\left(t_{0}\right) t+\frac{1}{2} u_{O_{j}, x} t^{2}, & t \leq t_{O_{j}, \text { stop }}, \\
s_{O_{j}, x}\left(t_{O_{j}, \text { stop }}\right), & t>t_{O_{j}, \text { stop }},\end{cases} \\
& t_{O_{j}, \text { stop }}=\mid \begin{array}{ll}
\frac{v_{O_{j}, x}\left(t_{0}\right)}{u_{O_{j}, x}} \mid, \\
\forall u_{O_{j}, x} \in \mathbb{R}^{\geq 0}: \quad s_{O_{j}, x}(t)=s_{O_{j}, x}\left(t_{0}\right)+v_{O_{j}, x}\left(t_{0}\right) t+\frac{1}{2} u_{O_{j}, x} t^{2},
\end{array}
\end{aligned}
$$

with longitudinal position $s_{O_{j}, x}$, velocity $v_{O_{j}, x}$, acceleration $u_{O_{j}, x}$, and stopping time $t_{O_{j}, \text { stop }}$.

\section{Design of Maneuver Templates}

Subsequently, we propose three exemplary maneuver templates for cooperative merging on highways in emergency situations. Further maneuver templates can be similarly constructed.

Definition 2 (Cooperative Merge (Three Vehicles)):

Initial Constraints: Let $\mathcal{V}=\left\{V_{1}, V_{2}, V_{3}\right\}$ be the set of vehicles which form a collaborative group and $\mathcal{O}=\left\{O_{1}\right\}$ be the set of obstacles. Vehicles $V_{2}$ and $V_{3}$ are situated in lane 1 , whereas vehicle $V_{1}$ and obstacle $O_{1}$ are located in lane 2 (see Fig. 3a). Lane 1 and lane 2 have identical driving directions. At initial time $t_{0}$, the longitudinal velocity of each cooperative vehicle is nonzero, and all vehicles follow the road course:

$$
\begin{array}{ll}
b_{1 / 2 / 3}\left(\mathbf{x}_{0}, t_{0}\right): & -v_{V_{1 / 2 / 3}, x}\left(t_{0}\right)<0, \\
b_{1 / 2 / 3}\left(\mathbf{y}_{0}, t_{0}\right): & v_{V_{1 / 2 / 3}, y}\left(t_{0}\right)=0 .
\end{array}
$$

Additionally, vehicle $V_{2}$ obeys the safe distance $s_{V_{3}, V_{2}}^{\mathrm{saf}}\left(t_{0}\right)$ :

$$
b_{4}\left(\mathbf{x}_{0}, t_{0}\right): \quad s_{V_{2}, x}\left(t_{0}\right)-s_{V_{3}, x}\left(t_{0}\right)+s_{V_{3}, V_{2}}^{\text {safe }}\left(t_{0}\right) \leq 0 .
$$

Obstacle $O_{1}$ is in front of vehicle $V_{1}$ which does not keep the safe distance (e.g. due to a cut-in of $O_{1}$ ):

$$
b_{5}\left(\mathbf{x}_{0}, t_{0}\right): \quad s_{O_{1}, x}\left(t_{0}\right)-s_{V_{1}, x}\left(t_{0}\right)-s_{O_{1}, V_{1}}^{\text {sae }}\left(t_{0}\right)<0 .
$$

State Constraints: Vehicles $V_{2}$ and $V_{3}$ follow the lane for all times:

$$
h_{1 / 2}(\mathbf{y}(t), t): \quad v_{V_{2 / 3}, y}(t)=0 .
$$

Vehicle $V_{1}$ and obstacle $O_{1}$ must not collide:

$$
h_{1}(\mathbf{x}(t), t): \quad s_{V_{1}, x}(t)-s_{O_{1}, x}(t) \leq 0 .
$$

The cooperative vehicles are not allowed to drive backwards:

$$
h_{2 / 3 / 4}(\mathbf{x}(t), t): \quad-v_{V_{1 / 2 / 3}, x}(t) \leq 0 .
$$

Terminal Constraints: Vehicle $V_{1}$ has covered a sufficient distance $w$ in lateral direction to lane 1 and moves tangentially to the road course:

$$
\begin{array}{ll}
a_{1}\left(\mathbf{y}\left(t_{f}\right), t_{f}\right): & s_{V_{1}, y}\left(t_{f}\right)-s_{V_{1}, y}\left(t_{0}\right)-w=0, \\
a_{2}\left(\mathbf{y}\left(t_{f}\right), t_{f}\right): & v_{V_{1}, y}\left(t_{f}\right)=0 .
\end{array}
$$

Vehicle $V_{1}$ is located in between vehicles $V_{2}$ and $V_{3}$, and all vehicles obey the safe distance:

$$
\begin{array}{ll}
a_{1}\left(\mathbf{x}\left(t_{f}\right), t_{f}\right): & s_{V_{2}, x}\left(t_{f}\right)-s_{V_{1}, x}\left(t_{f}\right) \leq 0, \\
a_{2}\left(\mathbf{x}\left(t_{f}\right), t_{f}\right): & s_{V_{2}, x}\left(t_{f}\right)-s_{V_{1}, x}\left(t_{f}\right)+s_{V_{1}, V_{2}}^{\text {safe }}\left(t_{f}\right) \leq 0, \\
a_{3}\left(\mathbf{x}\left(t_{f}\right), t_{f}\right): & s_{V_{1}, x}\left(t_{f}\right)-s_{V_{3}, x}\left(t_{f}\right) \leq 0, \\
a_{4}\left(\mathbf{x}\left(t_{f}\right), t_{f}\right): & s_{V_{1}, x}\left(t_{f}\right)-s_{V_{3}, x}\left(t_{f}\right)+s_{V_{3}, V_{1}}^{\text {safe }}\left(t_{f}\right) \leq 0 .
\end{array}
$$

Definition 3 (Cooperative Merge A (Two vehicles)): The traffic scene is the same as in Def. 2 with the exception that vehicle $V_{2}$ is missing (see Fig. $3 \mathrm{~b}$ ).

Definition 4 (Cooperative Merge B (Two vehicles)): The traffic scene is the same as in Def. 2 with the exception that vehicle $V_{3}$ is missing (see Fig. 3c). 


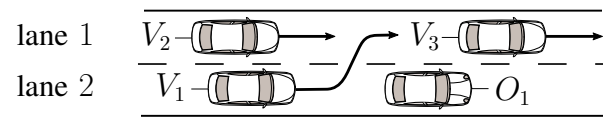

(a) Cooperative Merge (Three Vehicles)

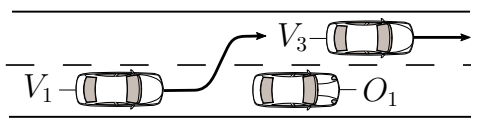

(b) Cooperative Merge A (Two Vehicles)

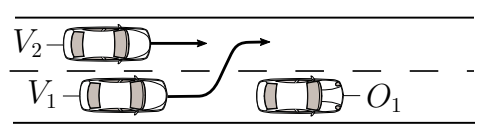

(c) Cooperative Merge B (Two Vehicles)

Fig. 3: Proposed maneuver templates for cooperative collision avoidance at highways.

Remark 1: Equations for the safe distance computation can be found in [22], [23] and depend on the vehicle order. In (8), it is assumed that vehicle $V_{1}$ is in front of vehicle $V_{2}$. However, (8) can be true although vehicle $V_{1}$ is behind vehicle $V_{2}$ due to the sign of $s_{V_{1}, V_{2}}^{\mathrm{safe}}\left(t_{f}\right)$. Therefore, we add (7). The same holds for (9) and (10).

Remark 2: It is possible to define a more comprehensive set of constraints for the proposed cooperative maneuvers, e.g. an upper velocity bound. However, the omission of constraints results in an overapproximation as stated in Sec. II.

Subsequently, we derive conditions to determine if $\mathcal{Z}(t)=$ $\emptyset$ holds for Def. 2. The conditions for Def. 3 and 4 can be deduced similarly, but fewer constraints have to be considered.

\section{Derivation of Solution Sets of Maneuver TEMPLATES}

In order to deduce if $\mathcal{Z}(t)=\emptyset$, we consider the following time-optimal control problem:

$$
\min t_{f}
$$

subject to

$$
\begin{gathered}
\dot{\mathbf{x}}(t)=\mathbf{A x}(t)+\mathbf{B} \mathbf{u}_{x}(t), \\
\underline{u}_{V_{m}, x} \leq u_{V_{m}, x}(t) \leq \bar{u}_{V_{m}, x}, \quad m \in\{1, \ldots, 3\}, \\
h_{j}(\mathbf{x}(t), t) \leq 0, \quad j \in\{1, \ldots, 4\}, \\
a_{i}\left(\mathbf{x}\left(t_{f}\right), t_{f}\right) \leq 0, \quad i \in\{1, \ldots, 4\}, \\
\dot{\mathbf{y}}(t)=\mathbf{A y}(t)+\mathbf{B} \mathbf{u}_{y}(t), \\
\underline{u}_{V_{m}, y} \leq u_{V_{m}, y}(t) \leq \bar{u}_{V_{m}, y}, \quad m \in\{1, \ldots, 3\}, \\
h_{k}(\mathbf{y}(t), t)=0, \quad k \in\{1,2\}, \\
a_{l}\left(\mathbf{y}\left(t_{f}\right), t_{f}\right)=0, \quad l \in\{1,2\} .
\end{gathered}
$$

We apply the minimum principle of Pontryagin to solve the optimal control problem (OCP) and refer to Hartl et al. [24] for a detailed survey of the maximum principle of Pontryagin. The following assumptions are made:

Assumption 1: The set of candidate solutions $\mathcal{K}(t)$ of the OCP (11a)-(11i), which is determined by the necessary first order optimality conditions, is $\mathcal{K}(t)=\emptyset$ if and only if the solution set $\mathcal{Z}(t)$ given by (11b)-(11i) is $\mathcal{Z}(t)=\emptyset$.

Assumption 2: Singular arcs are excluded [25].

Assumption 3: The entry times into a boundary arc and contact points differ for all state variable inequality constraints $h_{i}(\mathbf{x}(t), t)$.

Assumption 4: Every state variable inequality constraint $h_{i}(\mathbf{x}(t), t)$ is active during the optimization horizon $t \in$ $\left[t_{0}, t_{f}\right]$ once at most.

We exploit the fact that the dynamics of the vehicle model stated in Sec. IV is decoupled and solve the OCP in longitudinal and lateral direction separately. This means that the terminal times $t_{\text {lon }}$ and $t_{\text {lat }}$ are minimized until target sets (11e) and (11i) are reached. This yields the sets $\mathcal{K}_{\text {lon }}(t)$ and $\mathcal{K}_{\text {lat }}(t)$ of candidate solutions, respectively. The overall set of solutions $\mathcal{K}(t)$ is given by the Cartesian product of both sets $\mathcal{K}(t)=\mathcal{K}_{\text {lon }}(t) \times \mathcal{K}_{\text {lat }}(t)$. However, we have to consider that the terminal time $t_{f}$ of the entire OCP is given by $t_{f}=\max \left(t_{\text {lon }}, t_{\text {lat }}\right)$. Thus, the time-optimal solution is dominated by the slower motion. Subsequently, we account for this interdependency during the derivation of $\mathcal{K}_{\text {lon }}(t)$ and $\mathcal{K}_{\text {lat }}(t)$.

\section{A. Slower Motion: Lateral Motion}

The lateral lane-change maneuver of vehicle $V_{1}$ takes more time than the longitudinal gap adjustment, which means that $t_{f}=t_{\text {lat }}$.

a) Lateral Optimal Control: The set of constraints of the lateral OCP is composed of (11f)-(11i). Since the time has to be minimized, the cost function is

$$
J_{\text {lat }}=\int_{t_{0}}^{t_{\text {lat }}} 1 \mathrm{~d} t .
$$

Vehicle $V_{1}$ has to start the lateral lane-change maneuver at $t_{y}=t_{0}$.

b) Longitudinal Optimal Control: An OCP with fixed terminal time $t_{f}=t_{\text {lat }}$ is formulated. Vehicle $V_{1}$ should minimize its terminal position, and the gap between vehicles $V_{2}$ and $V_{3}$ should be maximized at terminal time $t_{f}$. A cost function in Mayer form [26] represents the objective:

$$
J_{\text {lon }}=s_{V_{1}, x}\left(t_{f}\right)+s_{V_{2}, x}\left(t_{f}\right)-s_{V_{3}, x}\left(t_{f}\right) .
$$

\section{B. Slower Motion: Longitudinal Motion}

In this case, the longitudinal gap adjustment takes more time than the lateral lane-change maneuver of vehicle $V_{1}$, which means that $t_{f}=t_{\text {lon }}$.

a) Lateral Optimal Control: We formulate the same lateral OCP as in Sec. VI-A. However, in order to maximize the time period for adjusting the gap between vehicles $V_{2}$ and $V_{3}$, vehicle $V_{1}$ is supposed to start the evasive maneuver as late as possible. Thus, the time $t_{y}$ to start the lane change is given by $t_{y}=t_{f}-t_{\text {lat }}$.

b) Longitudinal Optimal Control: We formulate a free terminal time problem, where the objective function minimizes $t_{f}=t_{\text {lon }}$ :

$$
J_{\text {lon }}=\int_{t_{0}}^{t_{f}} 1 \mathrm{~d} t .
$$

\section{Solution of the Optimal Control Problem}

The solution of the lateral and longitudinal OCPs stated in Sec. VI-A-VI-B, is given by Theorems 1-2, respectively. 
Theorem 1: Consider the OCP with constraints (11f)-(11i) and cost function (12). The set $\mathcal{K}_{\text {lat }}(t)$ can be obtained by the following control policies of vehicles $V_{1}, V_{2}$ and $V_{3}$ :

$$
\begin{aligned}
\forall t \in\left[t_{0}, t_{f}\right]: u_{V_{1}, y}(t) \begin{cases}=0 & t \in\left[t_{0}, t_{y}\right], \\
\in\left\{\underline{u}_{V_{1}, y}, \bar{u}_{V_{1}, y}\right\} & t \in\left[t_{y}, t_{f}\right],\end{cases} \\
u_{V_{2}, y}(t)=0 \\
u_{V_{3}, y}(t)=0
\end{aligned}
$$

where $u_{V_{1}, y}(t)$ is a bang-bang control for $t \in\left[t_{y}, t_{f}\right]$, which switches at most once at $t_{s, y} \in\left[t_{y}, t_{f}\right]$.

Proof: From (11h), it follows that $u_{V_{2}, y}(t)=0$ and $u_{V_{3}, y}(t)=0$. The time-optimal lane change maneuver of vehicle $V_{1}$ can be achieved by a bang-bang control [26].

Theorem 2: Consider the OCP with constraints (11b)(11e) and either cost function (13) or (14). Given that assumptions $2-4$ hold, the set $\mathcal{K}_{\text {lon }}(t)$ can be obtained by the following control policies of vehicles $V_{1}, V_{2}$ and $V_{3}$ :

$$
\begin{aligned}
\forall t \in\left[t_{0}, t_{f}\right]: & u_{V_{1}, x}(t) \in\left\{\underline{u}_{V_{1}, x}, \bar{u}_{V_{1}, x}\right\} \\
& u_{V_{2}, x}(t)= \begin{cases}\underline{u}_{V_{2}, x} & v_{V_{2}, x}(t)>0 \\
0 & v_{V_{2}, x}(t)=0\end{cases} \\
& u_{V_{3}, x}(t)=\bar{u}_{V_{3}, x},
\end{aligned}
$$

where $u_{V_{1}, x}(t)$ is a bang-bang control which switches at most once at $t_{s, x} \in\left[t_{0}, t_{f}\right]$.

Proof: The proof is based on Lemmas given in the Appendix.

Remark 3: It should be noted that collisions between vehicles $V_{2}$ and $V_{3}$ can be ruled out, since both vehicles obey the safe distance at the beginning of the maneuver (see (4)) and the preceding vehicle $V_{3}$ constantly accelerates, whereas the following vehicle $V_{2}$ constantly decelerates.

In summary, we have defined three maneuver templates (see Def. 2-4), and derived conditions to determine if $\mathcal{Z}(t)$ is empty. In the next section, a fast feasibility check of the maneuver templates based on a greedy algorithm is presented.

\section{Feasibility Check of Maneuver Templates}

Given the current traffic situation, we aim to determine if $\mathcal{Z}(t)=\emptyset$ holds for a maneuver template. We show this by contradiction and try to find an input trajectory $\mathbf{u}(\cdot)$ such that all constraints $\mathcal{C}$ are fulfilled. According to Theorems $1-2$, it suffices to search switching times $t_{s, x}$ and $t_{s, y}$ of the longitudinal and lateral bang-bang control as well as a starting time $t_{y}$ of the lateral evasive maneuver of vehicle $V_{1}$ such that no constraint $C_{i} \in \mathcal{C}$ is violated.

According to [20], $t_{s, y}$ can be computed analytically for a given lateral displacement $w$ such that constraints (11i) hold. In order to search valid time instances $t_{s, x}$ and $t_{y}$, we use a greedy algorithm based on binary search [27]. As a byproduct, we obtain a reference trajectory $\mathbf{z}_{V_{n}}(\cdot)$ for each collaborative vehicle, which does not violate any constraint $C_{i} \in \mathcal{C}$, if such a reference trajectory exists.

\section{A. Greedy Algorithm}

A binary search works as follows [27]: It is assumed that the target value $t$ is within the interval $t \in[\underline{t}, \bar{t}]$, which is recursively adapted according to a predefined policy. We choose the new value $t$ as the bisector of the adjusted interval. The algorithm terminates if a solution is found or the difference $|\Delta t|$ of the value $t$ between two consecutive iterations is smaller than a threshold $\epsilon$.

Our feasibility check is based on two nested binary searches (see Alg. 1): In an outer loop, an admissible value $t_{s, x} \in\left[\underline{t}_{s, x}, \bar{t}_{s, x}\right]$ for a given bang-bang control sequence, e.g. first accelerate then decelerate, is searched. A binary search for a valid starting time $t_{y} \in\left[\underline{t}_{y}, \bar{t}_{y}\right]$ of the lateral movement of vehicle $V_{1}$ for the given longitudinal input trajectory $u_{V_{1}, x}(\cdot)$ is thereby recursively called (line 4$)$. If there does not exist an admissible time instance $t_{y}$ for the current input $u_{V_{1}, x}(\cdot)$ such that all constraints $\mathcal{C}$ are satisfied, the interval $\left[\underline{t}_{s, x}, \bar{t}_{s, x}\right]$ is updated. The policies for updating the intervals $\left[\underline{t}_{s, x}, \bar{t}_{s, x}\right]$ and $\left[\underline{t}_{y}, \bar{t}_{y}\right]$ are elaborated in the next subsection.

We repeat the procedure for every possible bang-bang control sequence until a solution is found. If none of the bang-bang control sequences result in a feasible trajectory $\mathbf{z}(\cdot)$, we assume that $\mathcal{Z}(t)=\emptyset$.

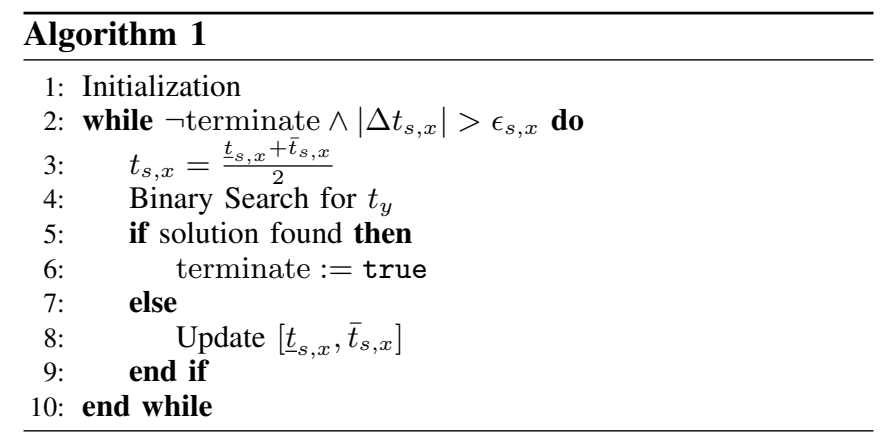

\section{B. Adjustment of Intervals}

The adjustment of the time intervals depends on the violated constraints $C_{i} \in \mathcal{C}$. The greedy algorithm allows us to easily incorporate the shapes of vehicles. Thus, we can reduce the overapproximation of $\mathcal{Z}(t)$ due to the assumption that the vehicles are point masses and replace constraint (6) with the restriction that the occupancy $\mathcal{Q}_{V_{n}}(t)$ of each cooperative vehicle and obstacle $\mathcal{Q}_{O_{j}}(t)$ must not intersect.

We introduce the set $\mathcal{C}_{V_{2}}$, which comprises terminal constraints $a_{i}\left(\mathbf{x}\left(t_{f}\right), t_{f}\right)$, for $i \in\{1,2\}$, and constraint $\forall t$ : $\mathcal{Q}_{V_{1}}(t) \cap \mathcal{Q}_{V_{2}}(t)=\emptyset$. Similarly, we define the set $\mathcal{C}_{V_{3}}$ with terminal constraints $a_{i}\left(\mathbf{x}\left(t_{f}\right), t_{f}\right)$, for $i \in\{3,4\}$, and $\forall t: \mathcal{Q}_{V_{1}}(t) \cap \mathcal{Q}_{V_{3}}(t)=\emptyset$. Furthermore, we have $\mathcal{C}_{O_{1}}$ containing constraint $\forall t: \mathcal{Q}_{V_{1}}(t) \cap \mathcal{Q}_{O_{1}}(t)=\emptyset$.

1) Policy for $t_{y}$ : The interval $\left[\underline{t}_{y}, \bar{t}_{y}\right]$ is updated according to the following policy:

- Whenever a constraint $C_{i} \in \mathcal{C}_{O_{1}}$ is violated, vehicle $V_{1}$ must change lanes earlier: $\bar{t}_{y}=t_{y}$ (see Fig. 4b).

- Otherwise, if at least one constraint $C_{i} \in \mathcal{C}_{V_{2}} \cup \mathcal{C}_{V_{3}}$ is violated, vehicle $V_{1}$ must change lanes later: $\underline{t}_{y}=t_{y}$ (see Fig. 4a) 


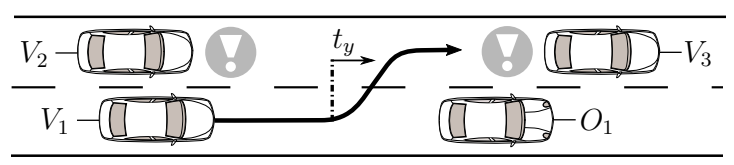

(a) Collision with $V_{2}$ or $V_{3} \Rightarrow$ increase $t_{y}$

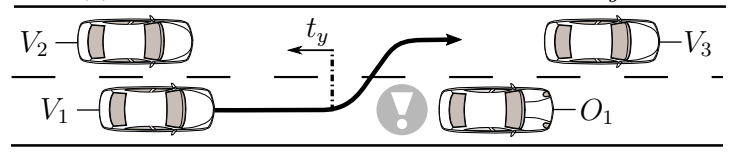

(b) Collision with $O_{1} \Rightarrow$ decrease $t_{y}$

Fig. 4: Update of interval $\left[\underline{t}_{y}, \bar{t}_{y}\right]$.

2) Policy for $t_{s, x}$ : The interval $\left[\underline{t}_{s, x}, \bar{t}_{s, x}\right]$ is adjusted based on the last evasive trajectory $\mathbf{z}_{V_{1}}$,last $(\cdot)$ for a given longitudinal input $u_{V_{1}, x}(\cdot)$ with respect to obstacle $O_{1}$, disregarding vehicles $V_{2}$ and $V_{3}$ :

- If $\mathbf{z}_{V_{1} \text {,last }}(\cdot)$ violates constraints $C_{i} \in \mathcal{C}_{V_{2}} \wedge C_{j} \in$ $\mathcal{C}_{V_{3}} \cup \mathcal{C}_{O_{1}}$, no solution exists.

- If there does not exist any trajectory $\mathbf{z}_{V_{1} \text {,last }}(\cdot)$ preventing a collision between $V_{1}$ and $O_{1}$, or if $\mathbf{z}_{V_{1}}$, last $(\cdot)$ violates constraints $C_{i} \in \mathcal{C}_{V_{3}}$, the deceleration period of the longitudinal bang-bang control is increased (see Fig. 5a).

- If $\mathbf{z}_{V_{1} \text {,last }}(\cdot)$ violates constraints $C_{i} \in \mathcal{C}_{V_{2}}$, the deceleration period of the longitudinal bang-bang control is decreased (see Fig. 5b).

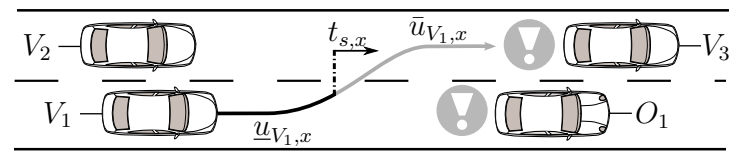

(a) Collision with $O_{1}$ or $V_{3} \Rightarrow$ increase time interval of $\underline{u}_{V_{1}}$,x

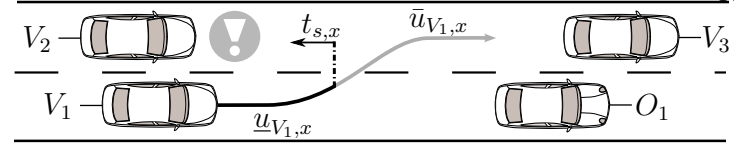

(b) Collision with $V_{2} \Rightarrow$ decrease time interval of $\underline{u}_{V_{1}, x}$

Fig. 5: Update of interval $\left[\underline{t}_{s, x}, \bar{t}_{s, x}\right]$.

\section{NUMERICAL EXAMPLES}

In this section, we evaluate the runtime of the feasibility check. Since we concentrate on emergency situations, it is important to quickly decide if a driving strategy is feasible. Therefore, we generate 100 scenarios denoted by $\mathcal{S}_{1}$ according to Def. 2 in order to analyze the performance of the maneuver template Cooperative Merge (Three Vehicles). The initial longitudinal position and velocity of each traffic participant are sampled uniformly. Furthermore, we generate sets $\mathcal{S}_{2}$ and $\mathcal{S}_{3}$ by removing vehicle $V_{2}$ or $V_{3}$ for all $S_{i} \in \mathcal{S}_{1}$ in order to evaluate maneuver templates Cooperative Merge A (Two Vehicles) and Cooperative Merge B (Two Vehicles), respectively. The simulation is performed on an $\operatorname{Intel}(\mathrm{R})$ Core(TM) i7-6700HQ $2.60 \mathrm{GHz}$ processor with $16 \mathrm{~GB}$ of RAM. The code is implemented in MATLAB R2016b.

Tab. I shows how often a maneuver template was evaluated as feasible and the median runtime over all scenarios $S_{i}$. Although the code is not optimized, the runtime of the

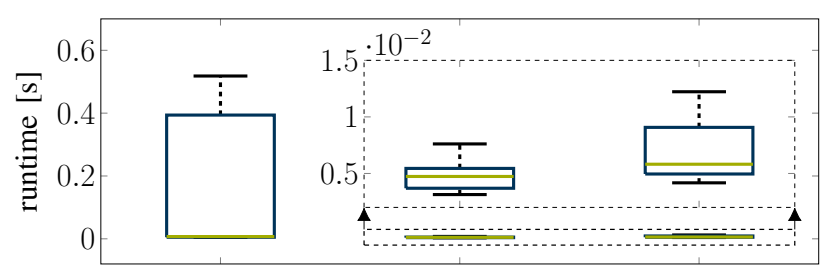

$\begin{array}{lll}\text { Def. } 2 \text { Def. } 3 & \text { Def. } 4\end{array}$

Fig. 6: Runtime of the feasibility check of the proposed maneuver templates according to Def. 2-4. The dashed box indicates a zoom of the boxplots for Def. 3-4.

TABLE I: Evaluation of the feasibility check.

\begin{tabular}{lrc}
\hline Maneuver Template & Feasible & Time [ms] \\
\hline Cooperative Merge (Three Vehicles) & 68 & 7.2 \\
Cooperative Merge A (Two Vehicles) & 91 & 4.7 \\
Cooperative Merge B (Two Vehicles) & 77 & 5.8 \\
\hline
\end{tabular}

feasibility check of all proposed maneuver templates is on average (median) less than $10 \mathrm{~ms}$. Fig. 6 shows the boxplots of the runtime of all maneuver templates according to Def. 24. The 75 th percentile of maneuver template Cooperative Merge (Three Vehicles) is $0.39 \mathrm{~s}$, which is $\approx 54 \mathrm{x}$ the median runtime of this maneuver template. This is because in 32 scenarios of set $\mathcal{S}_{1}$, the maneuver template is not feasible. The binary search has to evaluate all possible bang-bang input sequences, which entails more collision checks, and thus, a higher runtime. However, since maneuver templates can be evaluated in parallel, we believe that this does not affect the applicability of our approach.

Subject to future research is the assessment of feasible maneuver templates and the incorporation of maneuver templates into a motion planner. The trajectories $\mathbf{z}_{V_{n}}(\cdot)$ of the maneuver templates can be used as a basis for computing the cost of a maneuver template and as an initial solution for the motion planner. Moreover, Alg. 1 can be modified: As soon as a feasible solution is found, it can be refined according to some cost function, similar to anytime algorithms (see Fig. 7).

\section{CONCLUSIONS}

We present a novel approach based on maneuver templates for selecting cooperative driving strategies. Through the utilization of maneuver templates, complex decision making in a group of collaborative vehicles is more predictable and transparent in contrast to learning-based methods. More importantly, we can show the infeasibility of certain maneuver templates for a given situation. This is useful in terms of the certifiability of safety-critical algorithms. We have

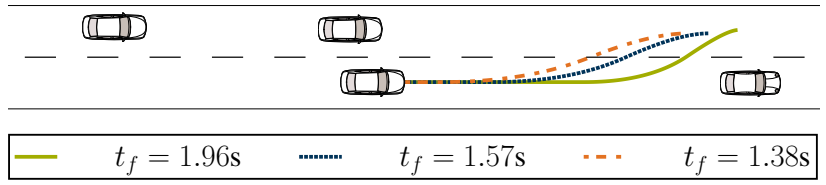

Fig. 7: Search for time-optimal trajectory $\mathbf{z}_{V_{1}}(\cdot)$. 
demonstrated that the computation of our proposed maneuver templates is computationally efficient, although we consider the full set of possible actions for each cooperative vehicle. In the future, we plan to investigate how many maneuver templates are necessary to cover the majority of dangerous traffic situations. We will thereby analyze different methods to derive maneuver templates.

\section{APPENDIX}

To prove Theorem 2, we split its proof into 6 Lemmas, and use the minimum principle of Pontryagin (indirect adjoining approach). Combining all Lemmas, we can show the following: Vehicle $V_{1}$ does not stop during the maneuver, since the state constraint $h_{2}(\mathbf{x}(t), t)$ is inactive for $t \in\left[t_{0}, t_{f}\right]$ (see Lemma 2). Furthermore, $h_{1}(\mathbf{x}(t), t)<0$ for $t \in\left[t_{0}, t_{f}[\right.$ and $h_{1}\left(\mathbf{x}\left(t_{f}\right), t_{f}\right) \leq 0$ hold, meaning that the distance between vehicle $V_{1}$ and obstacle $O_{1}$ can only vanish at terminal time $t_{f}$ (see Lemmas 2-3). However, this does not affect the solution of the unconstrained OCP of vehicle $V_{1}$, which is a bang-bang control (see Lemma 1). Vehicle $V_{2}$ decelerates until it stops (see Lemmas 1, 4, and 5). State constraint $h_{4}(\mathbf{x}(t), t)$ is always inactive for vehicle $V_{3}$ (see Lemma 6); thus, we only have to consider the solution of the unconstrained OCP, where vehicle $V_{3}$ constantly accelerates (see Lemma 1).

Let us first introduce some preliminaries for Lemma 1-6. The Hamiltonian $H$ is defined as

$$
\begin{aligned}
H= & \lambda_{0} F(\mathbf{x}(t), t)+\lambda_{1}(t) x_{2}(t)+\lambda_{2}(t) u_{V_{1}, x}(t)+\lambda_{3}(t) x_{4}(t) \\
& +\lambda_{4}(t) u_{V_{2}, x}(t)+\lambda_{5}(t) x_{6}(t)+\lambda_{6}(t) u_{V_{3}, x}(t)
\end{aligned}
$$

with costates $\boldsymbol{\lambda}(t)=\left[\lambda_{1}(t), \ldots, \lambda_{6}(t)\right]^{T}$ and the constant $\lambda_{0} \geq 0$. From the first order conditions for optimality, the costates $\boldsymbol{\lambda}(t)$ can be directly calculated [24]

$$
\dot{\boldsymbol{\lambda}}(t)=\left[\begin{array}{c}
0 \\
-\lambda_{1}(t) \\
0 \\
-\lambda_{3}(t) \\
0 \\
-\lambda_{5}(t)
\end{array}\right] \Rightarrow \boldsymbol{\lambda}(t)=\left[\begin{array}{c}
p_{1} \\
p_{2}-p_{1} t \\
p_{3} \\
p_{4}-p_{3} t \\
p_{5} \\
p_{6}-p_{5} t
\end{array}\right]
$$

where $p_{1}, \ldots, p_{6}$ are parameters. The transversality condition defines the costate $\boldsymbol{\lambda}(t)$ at terminal time $t_{f}$ as [24]

$$
\begin{aligned}
\boldsymbol{\lambda}\left(t_{f}\right)=\lambda_{0} \frac{\partial S\left(\mathbf{x}^{*}\left(t_{f}\right), t_{f}\right)}{\partial \mathbf{x}} & +\sum_{i=1}^{4} \alpha_{i} \frac{\partial a_{i}\left(\mathbf{x}^{*}\left(t_{f}\right), t_{f}\right)}{\partial \mathbf{x}} \\
& +\sum_{j=1}^{4} \gamma_{j} \frac{\partial h_{j}\left(\mathbf{x}^{*}\left(t_{f}\right), t_{f}\right)}{\partial \mathbf{x}}
\end{aligned}
$$

with optimal state trajectory $\mathrm{x}^{*}$ and constant multipliers $\alpha_{i}, \gamma_{j}$ with the following properties

$$
\begin{array}{ll}
\alpha_{i} \geq 0, & \alpha_{i} a_{i}\left(\mathbf{x}^{*}\left(t_{f}\right), t_{f}\right)=0, \quad i \in\{1, \ldots, 4\} \\
\gamma_{j} \geq 0, & \gamma_{j} h_{j}\left(\mathbf{x}^{*}\left(t_{f}\right), t_{f}\right)=0, \quad j \in\{1, \ldots, 4\} .
\end{array}
$$

Lemma 1: Consider the constraints (11b)-(11e) and either cost function (13) or (14) and assume that $h_{j}(\mathbf{x}(t), t)<0$, for $j=1, \ldots, 4$ and $t \in\left[t_{0}, t_{f}\right]$ hold. The set $\mathcal{K}_{\text {lon }}(t)$ can be determined with the following control policies of vehicles $V_{1}, V_{2}$ and $V_{3}$ :

$$
\begin{aligned}
\forall t \in\left[t_{0}, t_{f}\right]: \quad & u_{V_{1}, x}(t) \in\left\{\underline{u}_{V_{1}, x}, \bar{u}_{V_{1}, x}\right\}, \\
& u_{V_{2}, x}(t)=\underline{u}_{V_{2}, x}, \\
& u_{V_{3}, x}(t)=\bar{u}_{V_{3}, x},
\end{aligned}
$$

where $u_{V_{1}, x}(t)$ is a bang-bang control which switches at most once at $t_{s, x} \in\left[t_{0}, t_{f}\right]$.

Proof: From (15) and (16), we can determine $p_{1}, \ldots, p_{6}$ :

$$
\boldsymbol{\lambda}(t)=\left[\begin{array}{c}
\alpha_{4}+\alpha_{3}-\alpha_{1}-\alpha_{2}+\lambda_{0} k_{0} \\
\alpha_{4} k_{3}-\alpha_{2} k_{1}+\lambda_{1}\left(t_{f}-t\right) \\
\alpha_{1}+\alpha_{2}+\lambda_{0} k_{0} \\
\alpha_{2} k_{2}+\lambda_{3}\left(t_{f}-t\right) \\
-\alpha_{3}-\alpha_{4}-\lambda_{0} k_{0} \\
-\alpha_{4} k_{4}+\lambda_{5}\left(t_{f}-t\right)
\end{array}\right]
$$

with

$$
k_{0}=\left\{\begin{array}{l}
1, \quad t_{f}=t_{\text {lat }} \\
0, \quad t_{f}=t_{\text {lon }}
\end{array}, \quad \forall l \in\{1, \ldots, 4\}: k_{l} \geq 0 .\right.
$$

The coefficients $k_{1}, \ldots, k_{4}$ result from the derivation of (8) and (10) with respect to $\mathbf{x}$ (see (16)), respectively, and depend on $v_{V_{n}, x}\left(t_{f}\right)$ and $\underline{u}_{V_{n}, x}$ for $n \in\{1,2,3\}$. The optimal control input can be deduced from

$$
\frac{\partial H}{\partial u_{V_{1}, x}}=\lambda_{2}(t), \frac{\partial H}{\partial u_{V_{2}, x}}=\lambda_{4}(t)>0, \frac{\partial H}{\partial u_{V_{3}, x}}=\lambda_{6}(t)<0 .
$$

Remark 4: Although singular arcs are excluded for the time-optimal control, the inputs of vehicles $V_{2}$ and $V_{3}$ may be undetermined because the terminal inequalities concerning vehicle $V_{2}$ or $V_{3}$ are inactive. This means $i \in\{1,2\}$ : $a_{i}\left(\mathbf{x}\left(t_{f}\right), t_{f}\right)<0$ or $i \in\{3,4\}: a_{i}\left(\mathbf{x}\left(t_{f}\right), t_{f}\right)<0$. Basically, this implies that the control input can be arbitrary as long as it does not violate any terminal constraint. Thus, the optimization problem is not unique anymore. However, regarding the specific application, we can state that feasible solutions are not excluded if vehicle $V_{2}$ decelerates and vehicle $V_{3}$ accelerates constantly. The same reasoning holds for vehicle $V_{1}$.

Lemma 2: If vehicle $V_{1}$ cannot avoid a collision with obstacle $O_{1}$ by braking, $h_{1}(\mathbf{x}(t), t)<0$ holds for $t \in\left[t_{0}, t_{f}[\right.$ and $h_{2}(\mathbf{x}(t), t)<0$ for $t \in\left[t_{0}, t_{f}\right]$.

Proof: If emergency braking does not prevent an accident between vehicle $V_{1}$ and obstacle $O_{1}$ (see (5)), vehicle $V_{1}$ cannot approach obstacle $O_{1}$, meaning $s_{V_{1}, x}(t)=s_{O_{1}, x}(t)$, without violating constraint $h_{1}(\mathbf{x}(t), t)$, since $v_{V_{1}, x}(t)>$ $v_{O_{1}, x}(t)$. This further implies that vehicle $V_{1}$ cannot stop during $t \in\left[t_{0}, t_{f}\right]$. Thus, $v_{V_{1}, x}(t)>0$ for $t \in\left[t_{0}, t_{f}\right]$. Solely at terminal time $t_{f}$, the longitudinal position of vehicle $V_{1}$ and obstacle $O_{1}$ can coincide, since the clearance in lateral direction is sufficient.

Lemma 3: Lemma 1 still holds for $h_{1}(\mathbf{x}(t), t)<0$ for $t \in\left[t_{0}, t_{f}\left[\right.\right.$ and $h_{1}\left(\mathbf{x}\left(t_{f}\right), t_{f}\right)=0$.

Proof: From (16), (17) and (18), we have $\hat{\lambda}_{1}(t)=$ $\lambda_{1}(t)+\gamma_{1}$ and $\hat{\lambda}_{2}(t)=\lambda_{2}(t)+\gamma_{1}\left(t_{f}-t\right)$. Hence, the costate 
$\hat{\lambda}_{2}(t)$ determining the optimal control of vehicle $V_{1}$ is still an affine function yielding a bang-bang control.

Lemma 4: The state inequality constraints $h_{j}(\mathbf{x}(t), t) \leq$ 0 for $j=2, \ldots, 4$ are only active on non-vanishing time intervals $t \in\left[\tau_{1}, \tau_{2}\right]$, with $\tau_{1}<\tau_{2}$.

Proof: We prove Lemma 4 by contradiction. If there exists an active contact point $\tau$, it lies on a bang-bang subarc (see Lemma 1). This implies that

$$
\begin{aligned}
& \forall i \in\{1,2,3\}: \quad u_{V_{i}, x}\left(\tau^{-}\right)=\underline{u}_{V_{i}, x}, \quad u_{V_{i}, x}\left(\tau^{+}\right)=\bar{u}_{V_{i}, x}, \\
& \lambda_{2 i}^{-}\left(\tau^{-}\right) \geq 0, \quad \lambda_{2 i}^{+}\left(\tau^{+}\right) \leq 0 .
\end{aligned}
$$

In order to satisfy the costate jump condition [24]

$$
\underbrace{\lambda_{2 i}^{-}\left(\tau^{-}\right)}_{\geq 0}=\underbrace{\lambda_{2 i}^{+}\left(\tau^{+}\right)}_{\leq 0}-\eta_{i}(\tau),
$$

we have $\eta_{i}(\tau) \leq 0$. However, $\eta_{i}(t)<0$ contradicts the sign condition of $\eta_{i}(t)$, which requires $\eta_{i}(t) \geq 0$. Thus, $\eta_{i}(\tau)=0$, which implies that the solution of the constrained and unconstrained OCP do not differ. This is contrary to the assumption that $\tau$ is an active contact point.

Lemma 5: Consider the constraints (11b)-(11e) and either cost function (13) or (14) and assume that $h_{3}(\mathbf{x}(t), t)=0$ holds for $t \in\left[\tau_{1}, \tau_{2}\right] \subset\left[t_{0}, t_{f}\right]$, with $\tau_{1}<\tau_{2}$. The control policy of vehicle $V_{2}$ is

$$
u_{V_{2}, x}(t)= \begin{cases}\underline{u}_{V_{2}, x}, & t \in\left[t_{0}, \tau_{1}^{-}\right] \\ 0, & t \in\left[\tau_{1}^{+}, t_{f}\right] .\end{cases}
$$

Proof: As long as the state inequality constraint $h_{3}(\mathbf{x}(t), t)$ is inactive, vehicle $V_{2}$ brakes. It is counterproductive if vehicle $V_{2}$ would accelerate. We can therefore conclude that vehicle $V_{2}$ remains steady once it has stopped. The optimal control along the boundary arc can be determined with

$$
\frac{\mathrm{d} h_{3}(\mathbf{x}(t), t)}{\mathrm{d} t}=0 \Rightarrow u_{V_{2}, x}(t)=0 .
$$

Lemma 6: State constraint $h_{4}(\mathbf{x}(t), t)$ is inactive for $t \in$ $\left[t_{0}, t_{f}\right]$.

Proof: Lemma 6 follows directly from (3), where we have $v_{V_{3}, x}\left(t_{0}\right)>0$, and Lemma 1 yields $u_{V_{3}, x}(t)>0$ for $t \in\left[t_{0}, t_{f}\right]$.

\section{REFERENCES}

[1] E. Semsar-Kazerooni and J. Ploeg, "Interaction protocols for cooperative merging and lane reduction scenarios," in IEEE 18th International Conference on Intelligent Transportation Systems, pp. 1964-1970, 2015.

[2] M. Amoozadeh, H. Deng, C.-N. Chuah, H. M. Zhang, and D. Ghosal, "Platoon management with cooperative adaptive cruise control enabled by VANET," Vehicular Communications, vol. 2, no. 2, pp. 110 - 123, 2015.

[3] C. Frese and J. Beyerer, "A comparison of motion planning algorithms for cooperative collision avoidance of multiple cognitive automobiles," in IEEE Intelligent Vehicles Symposium, pp. 1156-1162, 2011.

[4] T. Schouwenaars, B. De Moor, E. Feron, and J. How, "Mixed integer programming for multi-vehicle path planning," in European Control Conference, pp. 2603-2608, 2001.

[5] J.-B. Tomas-Gabarron, E. Egea-Lopez, and J. Garcia-Haro, "Vehicular trajectory optimization for cooperative collision avoidance at high speeds," IEEE Transactions on Intelligent Transportation Systems, vol. 14, no. 4, pp. 1930-1941, 2013.
[6] C. Frese, "Cooperative motion planning using branch and bound methods," in Proc. of the 2009 Joint Workshop of Fraunhofer IOSB and Institute for Anthropomatics, Vision and Fusion Laboratory (J. Beyerer and M. Huber, eds.), no. IES-2009-13, pp. 187-201, KIT Scientific Publishing, 2009.

[7] C. Frese, J. Beyerer, and P. Zimmer, "Cooperation of cars and formation of cooperative groups," in IEEE Intelligent Vehicles Symposium, pp. 227-232, 2007.

[8] R. Hult, G. R. Campos, P. Falcone, and H. Wymeersch, "An approximate solution to the optimal coordination problem for autonomous vehicles at intersections," in American Control Conference, pp. 763 $768,2015$.

[9] A. Colombo and D. Del Vecchio, "Least restrictive supervisors for intersection collision avoidance: A scheduling approach," IEEE Transactions on Automatic Control, vol. 60, no. 6, pp. 1515-1527, 2015.

[10] A. Colombo and D. Del Vecchio, "Efficient algorithms for collision avoidance at intersections," in Proc. of the 15th ACM International Conference on Hybrid Systems: Computation and Control, pp. 145$154,2012$.

[11] H. Kowshik, D. Caveney, and P. R. Kumar, "Provable systemwide safety in intelligent intersections," IEEE Transactions on Vehicular Technology, vol. 60, no. 3, pp. 804-818, 2011.

[12] T. Awal, M. Murshed, and M. Ali, "An efficient cooperative lanechanging algorithm for sensor- and communication-enabled automated vehicles," in IEEE Intelligent Vehicles Symposium, pp. 1328-1333, 2015.

[13] L. Li, F.-Y. Wang, and Y. Zhang, "Cooperative driving at lane closures," in IEEE Intelligent Vehicles Symposium, pp. 1156-1161, 2007.

[14] H. Rewald and O. Stursberg, "Cooperation of autonomous vehicles using a hierarchy of auction-based and model-predictive control," in IEEE Intelligent Vehicles Symposium, pp. 1078-1084, 2016.

[15] W. Yang, Z. Zhiyong, Y. Jianhua, and G. Lifen, "Static game approach for solving lane-merging conflict between autonomous vehicles," in IEEE International Conference on Intelligent Transportation Engineering, pp. 53-57, 2016.

[16] M. Elhenawy, A. A. Elbery, A. A. Hassan, and H. A. Rakha, "An intersection game-theory-based traffic control algorithm in a connected vehicle environment," in IEEE 18th International Conference on Intelligent Transportation Systems, pp. 343-347, 2015.

[17] D. Carlino, S. D. Boyles, and P. Stone, "Auction-based autonomous intersection management," in 16th International IEEE Conference on Intelligent Transportation Systems, pp. 529-534, 2013.

[18] M. Düring and K. Lemmer, "Cooperative maneuver planning for cooperative driving," IEEE Intelligent Transportation Systems Magazine, vol. 8, no. 3, pp. 8-22, 2016.

[19] D. Lenz, T. Kessler, and A. Knoll, "Tactical cooperative planning for autonomous highway driving using Monte-Carlo tree search," in IEEE Intelligent Vehicles Symposium, pp. 447-453, 2016.

[20] M. Althoff, D. Heß, and F. Gambert, "Road occupancy prediction of traffic participants," in 16th International IEEE Conference on Intelligent Transportation Systems, pp. 99-105, 2013.

[21] H. Zhang, A. Geiger, and R. Urtasun, "Understanding high-level semantics by modeling traffic patterns," in Proc. of the IEEE International Conference on Computer Vision, pp. 3056-3063, 2013.

[22] M. Althoff and R. Lösch, "Can automated road vehicles harmonize with traffic flow while guaranteeing a safe distance?," in Proc. of the 19th International IEEE Conference on Intelligent Transportation Systems, pp. 485-491, 2016.

[23] B. H. Wilson, "How soon to brake and how hard to brake: Unified analysis of the envelope of opportunity for rear-end collision warnings," in Proc. of 17th International Technical Conference on the Enhanced Safety of Vehicles, vol. 47, 2001.

[24] R. F. Hartl, S. P. Sethi, and R. G. Vickson, "A survey of the maximum principles for optimal control problems with state constraints," SIAM review, vol. 37, no. 2, pp. 181-218, 1995.

[25] G. Fraser-Andrews, "Finding candidate singular optimal controls: A state of the art survey," Journal of Optimization Theory and Applications, vol. 60, no. 2, pp. 173-190, 1989.

[26] D. Liberzon, Calculus of variations and optimal control theory: a concise introduction. Princeton University Press, 2012.

[27] G. R. Wood, "The bisection method in higher dimensions," Mathematical Programming, vol. 55, no. 1, pp. 319-337, 1992. 